\title{
PROPAGATION OF SHADOW EFFECTS ON TYPICAL REMOTE SENSING APPLICATIONS IN FORESTRY
}

\author{
P. Kempeneers ${ }^{1}, K$. Vandekerkhove ${ }^{2}$ \\ ${ }^{1}$ VITO \\ Boeretang 200, $2400 \mathrm{Mol}$, Belgium \\ ${ }^{2}$ INBO \\ Kliniekstraat 25, 1070 Brussel, Belgium
}

\author{
${ }^{3}$ F. Devriendt, F. Van Coillie \\ ${ }^{3}$ Universiteit Gent \\ Faculty of Bioscience Engineering \\ Coupure Links 653, 9000 Gent, Belgium
}

\begin{abstract}
Airborne LiDAR and hyperspectral data were acquired over a broadleaved forest area in Belgium. Shadow fractions were calculated, based on Sun angles and a digital surface model derived from the LiDAR data. Pixels in the hyperspectral image were classified based on the shadow fractions to study the effect of shadow on canopy reflectance and how the effect propagated to typical remote sensing applications in forestry. As a first application, the photosynthetical reflectance index (PRI) was studied, which expresses the relative down-regulation of photosynthesis. A strong correlation ( $R^{2}=0.93$ ) was found between the shadow fraction and the PRI. The second application was a tree species classification problem. A measure for classification uncertainty (CU) was introduced, based on the Shannon entropy. It was shown that the majority of pixels with a low shadow fraction were classified with a lower uncertainty.
\end{abstract}

Index Terms - Mutual tree canopy shading, hyperspectral forest applications, LiDAR data

\section{INTRODUCTION}

Mutual shading of tree crowns influence the radiometric properties of forest canopy. Some authors have tried to compensation for shadow effects using spectral mixture analysis [1, 2] and empirical functions [3]. Others have used shadow information to derive structural parameters of forest $[4,5,6]$. The objective of this work was to study the effect of tree canopy shadows on the canopy reflectance and, more in particular, how the effect propagates to remote sensing forest applications.

A simple hill shade algorithm was implemented to calculate the shadow fractions per pixel in the airborne hyperspectral image. As input, a digital (canopy) surface model (DSM) was used that was derived from LiDAR data. In case no such data are available, shadow fractions can be estimated from the radiance values in the image itself. In [5] tree canopy shadows were detected with a threshold DN value in a IKONOS panchromatic image and canopy fractions were derived for a multispectral image at a coarser spatial resolution.

In this study, both airborne hyperspectral imagery and high density airborne LiDAR data were available. The shadow effect was studied in different ways. First, the effect on the hyperspectral reflectance of tree canopies was quantified. Then we studied how the effect was propagated to typical forestry applications based on remote sensing. Two applications were selected: a quantitative application using the photochemical reflectance index (PRI [7]) and a classification problem on tree species.

\section{STUDY AREA AND MATERIAL}

Experiments were performed in Wijnendale, a forest area in Belgium. LiDAR data was obtained from a TopoSys sensor Harrier 56 at full waveform. The study area was acquired in four different flight lines. The resulting point density was $13.81 \mathrm{~m}^{-2}$ with a point spacing of $0.27 \mathrm{~m}$ (using all returns). A DTM and DSM were derived at a spatial grid of $0.5 \mathrm{~m} \times$ $0.5 \mathrm{~m}$. The DSM was obtained by selecting the maximum of all pulse returns in each grid, followed by a morphological closing filter (circular kernel of size $3 \times 3$ pixels). For the DTM, non-ground features (e.g., trees) were removed from the LiDAR point cloud using a progressive morphological filter, as proposed in [8].

Hyperspectral data was acquired with the Airborne Prism Experiment (APEX), the newly developed imaging spectrometer by a Swiss-Belgian consortium on behalf of the European Space Agency (ESA). This sensor covers a wide spectral range, including the short wave infrared $(372-2498 \mathrm{~nm})$ in 300 spectral bands. The spatial resolution was $1.5 \mathrm{~m}$.

Radiance values were atmospherically corrected to top of canopy reflectance, based on the radiative transfer model MODTRAN4 [9]. Geometric correction was based on direct georeferencing [10]. 


\section{METHODS}

\subsection{Calculation of digital elevation models}

Modern airborne sensors provide point densities of 4 LiDAR points $\mathrm{m}^{-2}$ and more and can produce accurate DSMs at a spatial resolution $\left(R_{L}\right)$ that is typically equal or finer than that of most airborne hyperspectral imagery: $R_{L}<=R_{H}$. In this case study, the spatial resolution of the APEX hyperspectral image was $1.5 \mathrm{~m}$. The DSM was created using a grid of $0.5 \mathrm{~m}$. The spatial detail (resolution) of the shadow mask, derived from the DSM, was therefore finer than the hyperspectral image $\left(R_{H}=3 \times R_{L}\right)$.

A canopy height model (CHM) was also produced by subtracting a DSM from a DTM. To create a DTM non-ground features (e.g., trees) had to be removed from the LiDAR point cloud first. We used a progressive morphological filter for this purpose. As proposed in [8], the window size of the filter was gradually increased, using elevation difference thresholds to remove non-ground measurements. Due to the unique combination of the thresholds and window size in each step, ground data could be preserved in this process. Some holes (nonfilled grid cells) still remained in the filtered output. These pixels were interpolated in the final DTM raster grid using an inverse distance weighting to the filled grid cells. A four direction conic search was performed to find the respective filled grid cells for each hole.

\subsection{Calculation of sunlit and shadow tree crowns}

The mutual shading of forest canopies was expressed as a shadow fraction with a hillshade algorithm in each hyperspectral image pixel. We assumed clear sky conditions, which was the case at the time when hyperspectral image was acquired. Both the Sun zenith angle (sza) and Sun azimuth angle (saa), needed for the hillshade algorithm, were known. As suggested in [11], the shadow fraction is hereby likely to be overestimated due to the translucent character of canopies. However, for this study crown transparency was not taken into account. Although the assumption of opaque canopies is unrealistic, this simplified model served the objective in this study.

A schematic overview of the shadow detection method is shown in Fig. 1. A tree at pixel $\mathrm{p}$ has a height $\mathrm{h}$ and casts shadows along the line defined by the angle saa ${ }^{\prime}=s a a+\pi$, where saa is the Sun azimuth angle in pixel p. The length of the shadow cast on the ground (d) without the obstruction of another above ground target, is proportional to the cotangent of the Sun's elevation angle in point $\mathrm{p}^{\prime}\left(\mathrm{sza}^{\prime}\right)$. Due to the short distance of d (typically less than $100 \mathrm{~m}$ relative to the Sun, we can approximate sza' $\approx$ sza. The elevation at point (potentially another tree) $\mathrm{p}^{\prime}$ is represented by $h^{\prime}$. A binary shadow mask was obtained from the DSM image, with pixels classified as either sunlit or shaded, following algorithm 3.2.

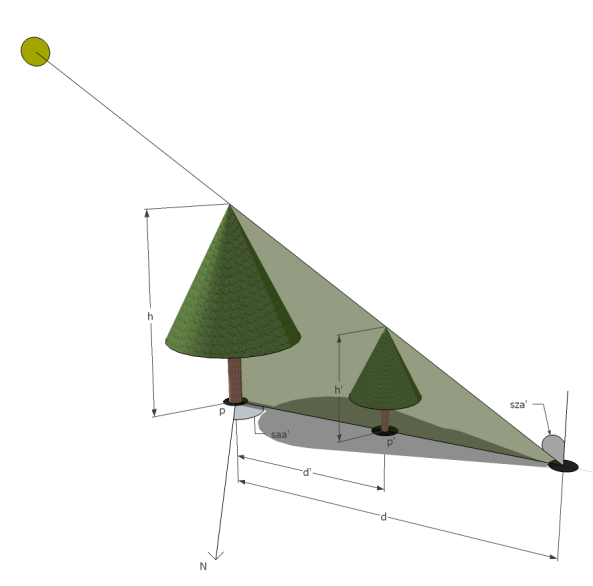

Fig. 1. Schematic representation of shadow cast from tree

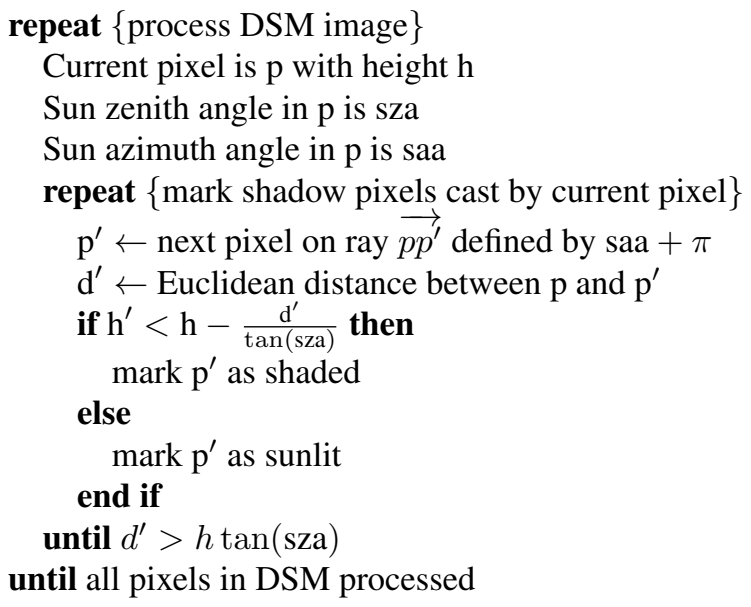

The CHM was used to create a binary tree canopy mask. We applied a simple height threshold equal to the lowest tree of interest $(20 \mathrm{~m})$. A binary tree canopy shadow mask $\mathrm{SHM}_{T}$ was then obtained by masking non-trees in the binary shadow mask. To obtain the shadow fraction map $\left(\mathrm{SHF}_{T}\right), \mathrm{SHM}_{T}$ was degraded to the spatial resolution of the hyperspectral image by calculating the proportion of shaded pixels that fell within the degraded pixel. With nine pixels in $\mathrm{SHM}_{T}$ for each hyperspectral pixel $\left(R_{H}=3 \times R_{L}\right)$, there were ten potential shadow fractions $(0,1 / 9,2 / 9, \ldots, 9 / 9)$.

\subsection{Impact of shadow effects on forest studies}

The impact of shadow effects on forest studies was studied at different levels. First, the impact on the canopy reflectance was quantified in general, based on the shadow fraction in the hyperspectral pixels. Then we quantified the propagation of the effect on typical forestry applications.

An important parameter used for quantitative studies in forestry is the photochemical reflectance index (PRI), a normalized difference spectral index that is based on two narrow $(3-10 \mathrm{~nm})$ wavelengths in the green spectrum. It expresses 
the relative down-regulation of photosynthesis induced primarily by high light intensities [7]:

$$
\mathrm{PRI}=\frac{\mathrm{R}_{531}-\mathrm{R}_{570}}{\mathrm{R}_{531}+\mathrm{R}_{570}}
$$

In contrast to some other vegetation indices that try to reduce illumination conditions, this index has a strong correlation to shadow fraction [12]. The PRI values are expected to increase with increasing shadow fractions.

The second case study was the classification of tree species in a broadleaved forest study area. The effect of canopy shadow fraction was assessed on the classification accuracy. However, traditional measures of classification accuracy such as overall accuracy and Kappa coefficient could not easily be adopted here, due to an insufficient test sample covering both sunlit and shaded pixels. Therefore, a different approach was followed. The classifier, a support vector machine, was trained to predict (continuous) probability estimates rather than (discrete) classes. The level of uncertainty was expressed (in bits) by the (Shannon) entropy (H):

$$
\mathrm{H}=-\sum_{i=1}^{C} P\left(\omega_{i}\right) \log _{2}\left(P\left(\omega_{i}\right)\right)
$$

with $P\left(\omega_{i}\right)$ the posterior probability for class $\omega_{i}(i=$ $1, \ldots, C)$ obtained from the classifier output. The classification uncertainty $(\mathrm{CU})$ was then rescaled as a percentage $(0-100 \%)$ as:

$$
\mathrm{CU}=-\frac{100}{\log _{2}(C)} \sum_{i=1}^{C} P\left(\omega_{i}\right) \log _{2}\left(P\left(\omega_{i}\right)\right)
$$

A spatially explicit measure of uncertainty was then obtained by calculating $\mathrm{CU}$ for each hyperspectral pixel. Using the shadow fraction cover map as an overlay, the classification uncertainty was studied in function of shadow fraction cover.

\section{RESULTS}

\subsection{Digital surface model and shadow mask}

A DSM was obtained from LiDAR data with a spatial resolution of $0.5 \mathrm{~m}$ as explained in section 3.1. The result is shown in Fig. 2(a). A binary shadow mask was then calculated following algorithm 3.2. The shadow fraction image, calculated at the spatial resolution of the hyperspectral image $(1.5 \mathrm{~m} \times 1.5 \mathrm{~m})$, is shown in Fig. 2(b).

\subsection{Shadow effect on canopy reflectance}

The effect of shadow on the canopy reflectance was studied by extracting a random sample $S_{\text {tr }}$ for each of the ten classes corresponding to the fraction of shadow cover. The respective mean reflectances are shown in Fig. 3. The canopy reflectance

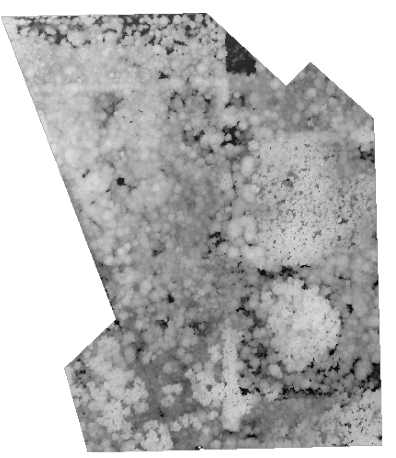

(a) Digital surface model

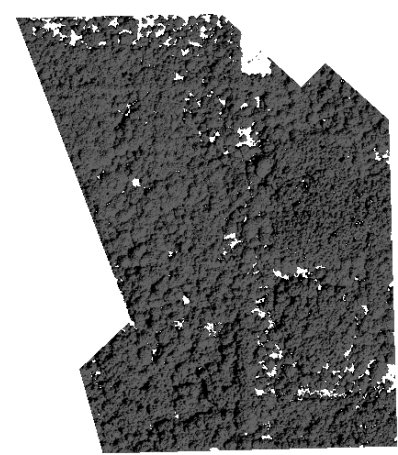

(b) Shadow fractions (dark pixels are more shaded
Fig. 2. DSM and shadow fraction

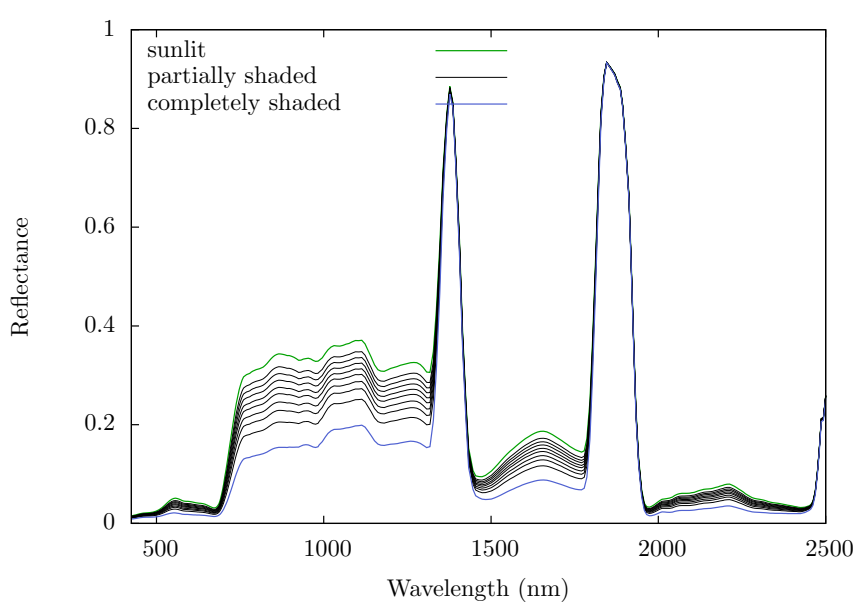

Fig. 3. Canopy reflectance spectra for different fractions of shadow.

of pixels that were fully shaded decreased up to $60 \%$ with respect to sunlit pixels. It was also shown that the reflectance strictly decreased for the different shadow fractions. The decrease was nearly linear with shadow fractions, but was wavelength dependent.

\subsection{Shadow effect on photochemical reflectance index}

The PRI was calculated for all tree canopy pixels. The respective means for each of the ten classes corresponding to the fraction of shadow cover are shown in Fig. 4. It was shown that PRI increases with increasing shadow fraction cover, which confirms conclusions in [12]. A strong correlation $\left(R^{2}=0.93\right)$ was found between the shadow fraction and the PRI index.

\subsection{Shadow effect on classification accuracy}

The effect of shadow on classification accuracy was studied for the Wijnendale forest area. The three main tree species 


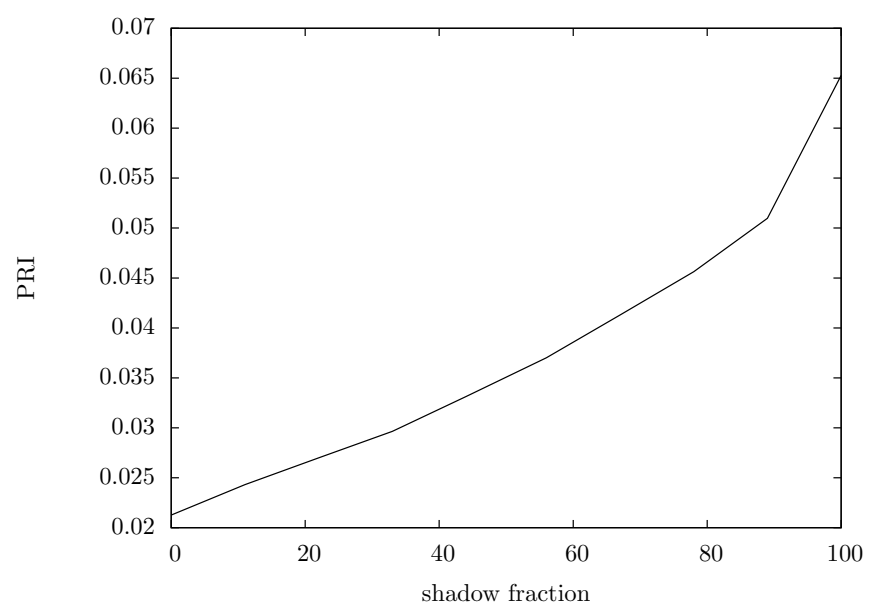

Fig. 4. PRI for different fractions of shadow
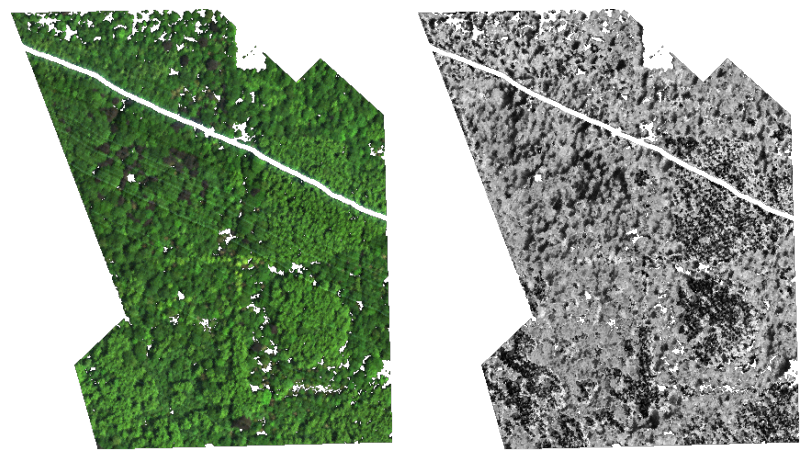

(a) APEX true color quicklook of (b) Classification uncertainty of tree the Wijnendale forest species mapping in Wijnendale forest. Bright pixels correspond to a high uncertainty.

Fig. 5. Classification of tree species

in that area were Beech (Fagus Sylvatica), Poplar (Populus $x$ euramericana nigra) and Pedunculate Oak (Quercus robur). The classifier (support vector machine) output contained posterior probability estimates for each class, which were used to calculate the classification uncertainty according to equation 3 (UC). Posterior probabilities that were similar for the three classes $\left(P\left(\omega_{1}\right) \approx P\left(\omega_{2}\right) \approx P\left(\omega_{3}\right)\right.$ resulted in a high uncertainty. Distinct values, with $P\left(\omega_{i}\right) \gg P\left(\omega_{j}\right), i \neq j$ resulted in a low uncertainty $(\approx 0 \%)$. The uncertainty for each pixel was calculated and is shown in Fig. 5(b).

The effect of shadow on the classification uncertainty (CU) is shown in Fig. 6. Some important observations can be made. Little pixels have a $100 \%$ uncertainty, corresponding to the case where the classifier output is identical for all three classes. This is only the case for extremely difficult classification problems (or poorly trained classifiers). The CU has a bi-modal shape showing maxima at $63 \%$ and $15 \%$. The maximum at $63 \%$ corresponds to the typical case where the

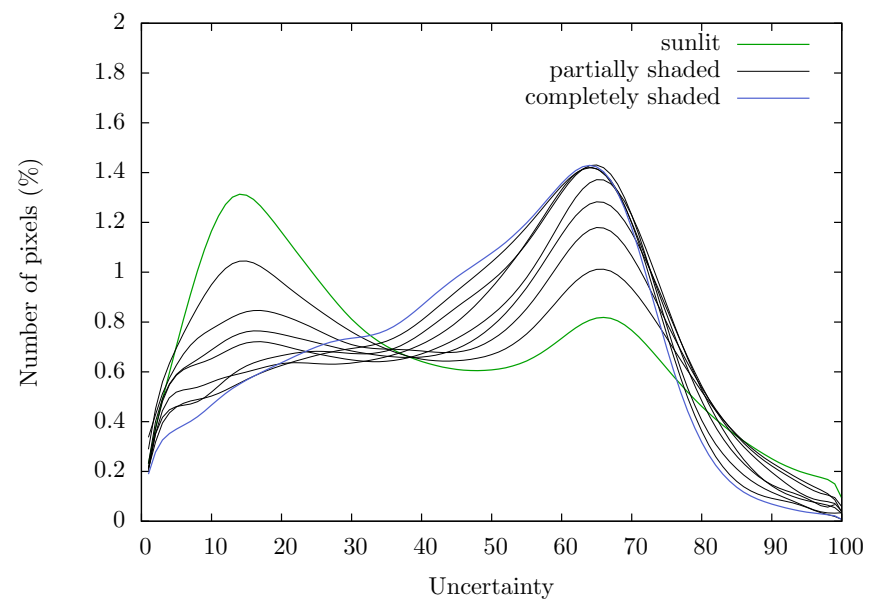

Fig. 6. Classification uncertainty in function of shadow fraction cover

posterior probability is high for two classes and low for one class. The ideal case when the posterior probability is $100 \%$ for only a single class corresponds to a CU of $0 \%(100 \%$ certain). The frequency of pixels for this ideal case is low but higher than $100 \%$ uncertainty. The second maximum corresponds to the more likely case where the posterior probability is high for a single class.

\section{CONCLUSION}

Mutual shading of tree crowns were found to have a large impact on remote sensing forest applications. The canopy reflectance of pixels that were fully shaded decreased up to $60 \%$ with respect to sunlit pixels. The decrease was nearly linear with shadow fractions, but was wavelength dependent.

The propagation of shadow effects to typical remote sensing applications in forestry was also studied. As a first application, the photosynthetical reflectance index (PRI) was studied, which expresses the relative down-regulation of photosynthesis. A strong correlation $\left(R^{2}=0.93\right)$ was found between the shadow fraction and the PRI. As a second application, a tree species classification problem was selected. It was shown that the majority of pixels with a larger shadow fraction were classified with a higher uncertainty. 


\section{REFERENCES}

[1] Derek R Peddle, Forrest G Hall, and Ellsworth F LeDrew, "Spectral mixture analysis and geometricoptical reflectance modeling of boreal forest biophysical structure," Remote Sensing of Environment, vol. 67, no. 3, pp. 288-297, 1999.

[2] Alan R Gillespie, Laura Gilson, MA Gillespie, and Van R Kane, "A framework for estimating unresolved spectral shade," in Second recent advances in quantitative remote sensing, j. A. Sobrino, Ed., 2006, pp. 385390.

[3] Van R Kane, Alan R Gillespie, Robert McGaughey, James A Lutz, Kevin Ceder, and Jerry F Franklin, "Interpretation and topographic compensation of conifer canopy self-shadowing," Remote sensing of Environment, vol. 112, no. 10, pp. 3820-3832, 2008.

[4] Warren B Cohen and Thomas A Spies, "Estimating structural attributes of douglas-fir/western hemlock forest stands from landsat and spot imagery," Remote Sensing of Environment, vol. 41, no. 1, pp. 1-17, 1992.

[5] G.P. Asner and A.S. Warner, "Canopy shadow in ikonos satellite observations of tropical forests and savannas," Remote Sensing of Environment, vol. 87, no. 4, pp. 521533, 2003.

[6] Jon Pasher and Douglas J King, "Multivariate forest structure modelling and mapping using high resolution airborne imagery and topographic information," Remote Sensing of Environment, vol. 114, no. 8, pp. 1718-1732, 2010.

[7] JA Gamon, J Penuelas, and CB Field, "A narrowwaveband spectral index that tracks diurnal changes in photosynthetic efficiency," Remote Sensing of Environment, vol. 41, no. 1, pp. 35-44, 1992.

[8] Keqi Zhang, Shu-Ching Chen, Dean Whitman, MeiLing Shyu, Jianhua Yan, and Chengcui Zhang, "A progressive morphological filter for removing nonground measurements from airborne lidar data," Geoscience and Remote Sensing, IEEE Transactions on, vol. 41, no. 4, pp. 872-882, 2003.

[9] A Berk, "Coauthors, 1999: Modtran-4 users manual," Air Force Research Laboratory, Space Vehicles Directorate, Air Force Materiel Command, Hanscom AFB, MA, 1999.

[10] K. M. Brown, G. M. Foody, and P. M. Atkinson, "Modelling geometric and misregistration error in airborne sensor data to enhance change detection," International Journal of Remote Sensing, vol. 28, pp. 2857-2879, 2007.
[11] T. Hilker, N.C. Coops, C.R. Schwalm, R.P.S. Jassal, T.A. Black, and P. Krishnan, "Effects of mutual shading of tree crowns on prediction of photosynthetic light-use efficiency in a coastal douglas-fir forest," Tree physiology, vol. 28, no. 6, pp. 825-834, 2008.

[12] F.G. Hall, T. Hilker, N.C. Coops, A. Lyapustin, K.F. Huemmrich, E. Middleton, H. Margolis, G. Drolet, and T.A. Black, "Multi-angle remote sensing of forest light use efficiency by observing pri variation with canopy shadow fraction," Remote Sensing of Environment, vol. 112, no. 7, pp. 3201-3211, 2008. 